\title{
The Analysis of Researches on Food That Reduce Stress: Vitamin C, Probiotics and Fermented foods
}

\author{
Elina Ziyu Jin ${ }^{1}$, Weiyan Zhang ${ }^{2}$, Puer Ding ${ }^{3 *}$

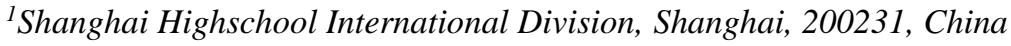 \\ ${ }^{2}$ The Stony Brook School, New York, NY11790, United States \\ ${ }^{3}$ School of Public Affairs Zhejiang University, Zhejiang, Hangzhou, 310058, China \\ ${ }^{*}$ Corresponding author. Email: $3180101665 @ z j u . e d u . c n$ \\ The authors for this paper have contributed the same
}

\begin{abstract}
This work accessed the credibility of the statements made in a media article published by CNN, titled "Food that Reduce Stress", by examining three clinical trials the media article derives from. Tools such as Cochrane ROB are implemented, and detailed analyses are made in assessing the presence of bias and potential problems within the methodology and presentation of result. Several common problems are found across the studies, including a limited sample size, the presence of selection bias, and the unclarity in trial designs. This work found that although all the statements correspond with the study outcomes, but the author of the media article has not mentioned any information about the quality of the studies he/she has cited.
\end{abstract}

Keywords: Food, Stress, Critical Analysis, Clinical Trial, Critique, Media Representation

\section{INTRODUCTION}

Stress has become an increasingly common problem in modern society, affecting millions of people worldwide.[1] Stress can have a range of negative consequences, including physical illness, emotional anxiety, and cognitive problems with memory and concentration. This not only affects people's daily life, but also further influences their physical and mental health if problems are not solved.[2] In addition, a study has shown that malnutrition can exacerbate anxiety [3].

Therefore, in addition to the traditional cognitive behavioral therapy and drug intervention, nutritional treatments for stress-related conditions also emerged. Related studies have also shown a relationship between improved nutrition and improved mental health outcomes, and a diet that includes fruits, vegetables, fish, olive oil may improve the mental condition.[4-5]

Under stress, it is important to eat healthy. A balanced and healthy diet is the key to helping human's bodies cope with the physiological changes caused by stress. More and more people are pursuing a more balanced diet to promote physical and mental health; Many media articles that focused on this topic are published recently.

Among them, an article named "Foods that can reduce stress" posted by CNN Health News stand out from the crowd. The article listed more than 10 types of food that can potentially alleviate anxiety. But are these foods provided in the article effective at reducing stress? In order to verify the credibility of the statements made in the article, this paper decided to closely inspect two statements about the effects of vitamin $\mathrm{C}$ and fermented foods on stress, and 3 studies (as shown in table 1) that the statements are based on. The majority of this paper is devoted to the critical analysis of the three journal articles from the perspectives of experimental design, sample selection, measurement method etc. In the end, this work will also make a judgment on the authenticity and reliability of the media articles, and finally put forward constructive suggestions. 
Table 1 Titles of the Journal Articles

A)Effects of Oral Vitamin C Supplementation on Anxiety in Students: A Double Blind, Randomized,

Placebo-Controlled Trial

B)A randomized controlled trial to test the effect of multispecies probiotics on cognitive reactivity to sad mood

C) Fermented foods, neuroticism, and social anxiety: An interaction model

\section{METHODOLOGY}

\subsection{Finding the Media Article}

The role of omega-3 fatty acids in preventing mood disorders, such as bipolar disorder, is gaining increasing attention So, this work decided to focus on this topic. The key words "effect of omega 3 fatty acid on mental health", and eventually found an article posted by CNN introducing the different types of food that can reduce stress.

\subsection{Locating the Journal articles}

This work found the journal articles through the direct links from the media article. Using studies that are cited by the media article ensures that the study results are highly relevant to the topic.

\subsection{Analyzing the Journal Articles}

This work deried a protocol for analyzing the journal articles, which is broken down into two general sections and a lot of specific steps. The protocol is located in section A of the annexes. The first general section is about how to take notes and extract information from the study, and the second general section is about organizing the information extracted into the tables that will be presented in the finding section. Each member follows this protocol to analyze their own journal article. The protocol is presented in the annexes section.

Moreover, this work used the cochrane risk of bias tool to assess the presence of bias in the two RCTs.

\section{FINDINGS}

\subsection{Critique of the Vitamin C Study}

This work has summarized the key features of the studies into tables that are presented in the annexes (sections B, C, and D). This works recommend you to refer to the tables while reading the following critiques.

The figure 1 below presents the risk of bias analysis of the 2 randomized control trials (the study about the vitamin $\mathrm{C}$ and the study about the probiotics) using the cochrane tool. More specific explanation of the risk analysis for each study will be presented in the corresponding critique section. The tool cannot be used to assess the presence of bias for the study about fermented foods, as it is an observational study instead of a RCT.

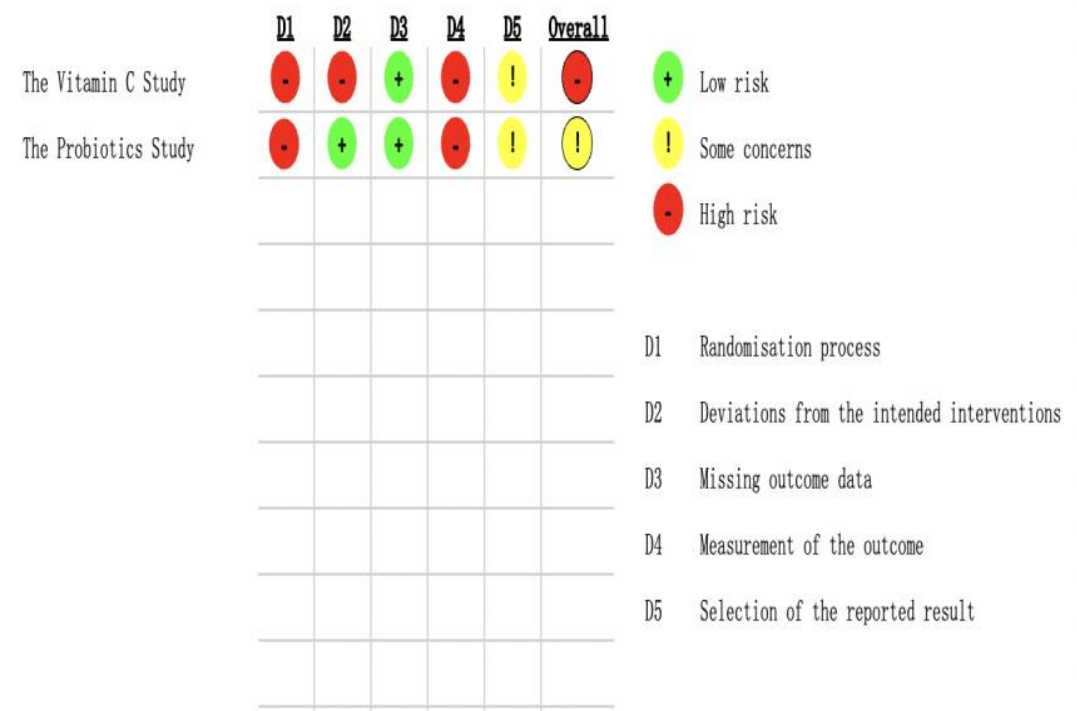

Figure 1 The risk of bias analysis of the 2 randomized control trials 


\subsubsection{Study Design}

\subsubsection{Size of the study}

The clinical trial is conducted on 42 high school students. However, no rationale about the selection of this sample size is included in the paper. The sample size is relatively small for a randomized, two-armed study. Moreover, the study did not provide any information about its power. Judging on the sample size, the power is unlikely to be high, which indicates a low chance for detecting a true effect.

\subsubsection{Inclusion Criteria}

The inclusion criteria are broad and unspecific. Firstly, the criterion of "good physical health" is prone to subjectivity, as the study did not include specific, quantifiable measures for its standard. One key inclusion criterion is the lack of a history of psychiatric conditions, which includes anxiety. This criterion should be emphasized as the study is attempting to investigate the effect of a treatment on the level of anxiety. However, the study did not include any description of how the students are assessed on their psychiatric condition in the pre-trial selection. This raises a problem.

Table 2 This is the table extracted from the result section of the study, showing the baseline BAI scores of the Vitamin $\mathrm{C}$ and the placebo control groups.[6]

\begin{tabular}{llllll}
\hline & \multicolumn{2}{c}{ Vitamin C group $(\mathrm{n}=21)$} & \multicolumn{2}{c}{ Placebo control group $(\mathrm{n}=21)$} & \\
Variables & Baseline & Post & Baseline & Post & p-value \\
\hline \multirow{2}{*}{ BAI scores } & $22.33 \pm 10.35$ & $16.86 \pm 10.19$ & $25.43 \pm 10.56$ & $24.95 \pm 13.11$ & 0.010
\end{tabular}

The main measurement for the level of anxiety in students the study used is the BAI test score. The BAI test is an inventory that measures the level of anxiety. Quoted from the study, "Scores between 8 and 15 are interpreted as mild anxiety, between 15 and 25 as moderate anxiety, and between 25 and 63 as severe anxiety". ${ }^{[7]}$ However, as shown in this table extracted from the study, the average baseline BAI score is 25.43 for the placebo control group and 22.23 for the treatment group, which indicate moderate to severe anxiety. These data contradict to the "no history of psychiatric condition" mentioned in the inclusion criteria. The contradiction reflects the possible invalidity of the inclusion criteria.

\section{$\underline{3.1 .1 .3 \text { baseline characteristics }}$}

Table 3 This is the table extracted from the result section of the study showing the baseline characteristics of the participants in the two groups [8]

\begin{tabular}{llll}
\hline Characteristics & Vitamin C & Placebo control & p-value \\
\hline Ages (years) & $30.43 \pm 14.35$ & $24.24 \pm 11.07$ & 0.125 \\
Sex N (female/male) & $21(16 / 5)$ & $21(16 / 5)$ & 1.000 \\
Height (m) & $1.62 \pm 0.09$ & $1.63 \pm 0.09$ & 0.930 \\
Weight $(\mathrm{kg})$ & $61.43 \pm 14.46$ & $59.00 \pm 11.59$ & 0.624 \\
BMI $\left(\mathrm{kg} \mathrm{m}^{-2}\right)$ & $23.32 \pm 4.77$ & $22.27 \pm 3.76$ & 0.513 \\
\hline
\end{tabular}

N: No. of participants, BMI: Body Mass Index, All values are Means \pm SDS, Student's-test, Chi-square test and Mann-Whitney U test

The baseline characteristics, shown in the above table, of each trial arm are assessed with student's t-test, and the study alleged no difference between the placebo group and the vitamin $\mathrm{C}$ group. It shows the study's intention to balance the initial conditions across the two groups to reduce selectional bias.
However, the study didn't mention on assessing one key characteristic----the baseline BAI scores. Judging from the data (the baseline BAI score for the vitamin C group is $22.33+-10.35$, while that of the placebo control group is $25.43+-10.56$ ), there seems to be a significant difference on the level of anxiety between the two groups. According to the BAI standard, a BAI score of 
22.33 indicates moderate anxiety, while a score of 25.43 already indicates severe anxiety. This piece of evidence shows that the baseline psychiatric conditions between the two groups are different, which can heavily influence the study result. Moreover, it seems to be unreasonable that the study used statistical tests to balance conditions like heights, BMI, and weight, which are less relevant to the focus of the study, instead of the baseline level of anxiety, the most important characteristic. The imbalance of the baseline characteristics has further implication in the quality of the study results presented later in the paper.

\subsubsection{Measurements of the Outcomes}

Besides from the BAI test score that is prone to subjectivity, the study also includes other, more objective measurements for its secondary outcomes, such as the heart rate and the systolic and diastolic blood pressure. However, these measurements do not reflect their level of anxiety due to the existence of multiple possible confounders. Vitamin C supplements may induce other physiological effects on the participants that may potentially lead to the changes in heart rate and blood pressures. Such physiological effects may not be related with the level of anxiety. The study recognized this shortcoming too. Even though it includes these measurements, but it did not specify that these measurements are direct reflections of the participants' level of anxiety. Instead, it clearly stated in the paper that the heart rate is a "parameter that is involved with anxiety symptom" [9].

There is a possible improvement in the study's design of measuring outcomes. The study should have considred including the blood cortisol level as one of its secondary outcomes. Blood cortisol level is a well-studied biomarker for stress related conditions, including anxiety. Its relationship to anxiety is more apparent than blood pressures and heart rate.

\subsubsection{Risk of biases}

The cochrane ROB tool signals the presence of selection and reporting bias in the study. I will analyze these 2 forms of bias in detail in the table 4 .

Table 4 Selection and reporting bias in studies (Cochrane ROB tool)

\begin{tabular}{|l|l|}
\hline $\begin{array}{l}\text { Type of Bias present in the } \\
\text { study }\end{array}$ & Explanation \\
\hline Selection Bias & $\begin{array}{l}\text { The study is randomized and doubly blinded, a design that elevates its objectivity } \\
\text { and reduces its selection bias. However, the way of randomization may not be } \\
\text { perfect to completely eliminate the imbalance among the treatment groups, as } \\
\text { seen in the imbalance of the baseline BAl scores across the placebo group and the } \\
\text { vitamin C group. No rationale about the randomization is presented in the study, } \\
\text { which further raise doubts to the effectiveness of this procedure. }\end{array}$ \\
\hline Reporting bias & $\begin{array}{l}\text { The level of anxiety in the trial is measured by the BAl test, a questionnaire } \\
\text { composed of } 21 \text { questions to assess the symptoms of anxiety. This tool of } \\
\text { measuring the final endpoint is prone to subjectivity, as the result is based on the } \\
\text { contestant's self-report. However, this source of bias is inevitable as the study wants } \\
\text { to measure the psychiatric condition of the participants, which always involves the } \\
\text { reflection of their subjective emotions. }\end{array}$ \\
\hline
\end{tabular}

\subsubsection{Presentation of the Results}

In presenting the effect of the treatments on the level of anxiety in students, the study mentioned two comparisons. These two comparisons are both problematic (Table 5 shows). 
Table 5 Analysis of two comparisons

\begin{tabular}{|c|c|}
\hline Comparisons in the Study & Why is it problematic \\
\hline $\begin{array}{l}\text { a) "...revealed that there was a significant } \\
\text { decrease [in mean BAl scores] among } \\
\text { vitamin C group post-treatment in } \\
\text { comparison to baseline treatment" }\end{array}$ & $\begin{array}{l}\text { In statement a), the study compares the baseline condition and the } \\
\text { post-treatment condition of the vitamin C group. However, a } \\
\text { meaningful comparison should be made between the two arms, but not } \\
\text { the before and after of a single arm. Thus, it is inappropriate for the } \\
\text { study to emphasize this statement as a major result. }\end{array}$ \\
\hline $\begin{array}{l}\text { b) "anxiety scores after the intervention } \\
\text { were significantly lower for the vitamin } \\
\mathrm{C} \text { than the placebo control group, } \\
\text { indicating that oral vitamin C } \\
\text { supplements improved the anxiety } \\
\text { levels of the student." }\end{array}$ & $\begin{array}{l}\text { In statement b), the study compares the after-treatment BAI score } \\
\text { between the placebo-control group and the vitamin C group. However, } \\
\text { this comparison is not meaningful as the baseline BAI scores between } \\
\text { the two groups are significantly different. Even before the intervention, } \\
\text { the study can still make such statement. A better statement can be made } \\
\text { from the comparison of the reduction in BAl scores between the two } \\
\text { groups. The result of this comparison should still be able to demonstrate } \\
\text { the better improvement in the vitamin C group's psychiatric condition } \\
\text { than that of the placebo group. However, such meaningful comparison } \\
\text { is not made in the study. }\end{array}$ \\
\hline
\end{tabular}

In general, the data the study collected are still meaningful and self-explanatory. However, the study failed to express the study results in a logically coherent way.

\subsubsection{Strengths and Limitations}

Finally, the work will summarize the limitation and strength of the study in the following table 6:

Table 6 Limitation and Strength

\begin{tabular}{|l|l|}
\hline \multirow{3}{*}{ Strength } & $\begin{array}{l}\text { - The study is randomized and double blinded to minimize selectional bias } \\
\text {-the study did generate a meaningful result that shows the effectiveness of vitamin C } \\
\text { supplements on alleviating anxiety } \\
\text { - relevant scientific literatures were discussed in the "Discussion" section of the study to show the } \\
\text { study results' alignment with previous studies. } \\
\text {-No missing data during the trial }\end{array}$ \\
\hline Limitations & $\begin{array}{l}\text { - presence of selection and reporting bias } \\
\text { - poorly written explanation of the results } \\
\text {-unclear methodology about pre-trial selection } \\
\text { epossible invalidity of the inclusion and exclusion criterion }\end{array}$ \\
\hline
\end{tabular}

\subsection{Critique of Critique of the Probiotics Study}

\subsubsection{Study Design}

\subsubsection{Size of the study}

This clinical trial includes a total of 40 participants $(20: 20)^{[10]}$, which is a very limited size for a randomized, two-armed study. The study did not provide any information on its power or its rationale for selection. This might indicate that the study result is to a large extent prone to ramdomness and not conclusive enough.

\subsubsection{Inclusion and Exclusion Criteria}

This study presents a quantitative, practiceable inclusion criteria. In the Participants section, they provided specific guidelines of inclusion and exclusion 
that they endorsed to rule out the health factors which would potentially affect the participants' response. The study included a pre-intervention screening process using the Mini International Neuropsychiatric Interview ${ }^{[11]}$, so that they ensure their participant group is strictly non-clinical and appropriate to their standards.

\subsubsection{Randomization}

This journal article lack any rationale on how they recruited the participants and how the sample was randomized. Based on its limited sample size, it could be deducted that their selected sample is not widely representable. Within their sample, there is also an uneven distribution of gender, which indicates selection bias. According to their provided information, their sample is predominantly female, which is another disadvantage brought by their small size of sample.

For randomization across the arms, the researches did include a statistical analysis that ensures no significant difference of gender distribution, BMI and age between the two groups (placebo vs intervention). This practice secures the power of their randomization within the sample.
The outcomes of this study are measured solely by surrogate means. They employed three questionnaires, the LEIDS-r, the Beck Depression Inventory II (BDI-II) and The Beck Anxiety Inventory (BAI), which are all self-reported and largely prone to subjectivity. All three questionnaires are provided and scored before and after the intervention. The LEIDS-r questionnaires, which access cognitive vulnerability on six aspects, is used as a means to ensure the consistency of the participants' psychological conditions. The latter two are both symptom based and are common in clinical diagnosis for depression and anxiety, which suggests that their measurement for non-clinical subects such as the participants in this study might not be very adequate. Only the changes in BDI-II score and the BAI score are being compared across the placebo and the intervention group. Therefore, their measurements of outcome lacks objectivity and could not accurately reflect the actual effect of their intervention.

\subsubsection{Risk of biases}

The cochrane ROB tool signals the presence of selection and reporting bias in the study (Table 7 shows)

\subsubsection{Measurements}

Table 7 The Presence of Selection and Reporting Bias

\begin{tabular}{|l|l|}
\hline Type of bias & Explanation \\
\hline Report bias & $\begin{array}{l}\text { The outcomes of the study are measured solely by questionnaire scores, which are largely prone } \\
\text { to subjectivity. The questionnaires they employed are also not fully appropriate to their } \\
\text { non-clinical purpose by design. }\end{array}$ \\
\hline Selection bias & $\begin{array}{l}\text { The article is randomized, controlled and triple blinded, and statistical analysis shown no } \\
\text { significant difference of characteristics across the arms. However, no rationale was provided on } \\
\text { how they selected the sample group. Moreover, the participant group is predominantly female, } \\
\text { which indicates a lack in universality. }\end{array}$ \\
\hline
\end{tabular}

\subsubsection{Strengtens and Limitations (Table 8 shows)}

Table 8 Limitation and Strength

\begin{tabular}{|l|l|}
\hline Strengthens & $\begin{array}{l}\text { - The study includes rigorous examination and confirmation, for example, pre-intervention } \\
\text { screening and bacteria sequencing } \\
\text { - careful with controlling baselines (additional BDI BAI scores) } \\
\text { - biological explanation of the results } \\
\text { - claimed as one of the first in probiotics vs mood } \\
\text { - Triple blind, randomized } \\
\text { - transparent data and analysis } \\
\text { - Stated no conflict of interest }\end{array}$ \\
\hline
\end{tabular}




\begin{tabular}{|l|l|}
\hline \multirow{2}{*}{ Limitations } & - Very imited sample size \\
- Selection bias: predominantly female \\
— Study design can not exclude probiotic intake outside of lab \\
•No confirmation of participants' daily intake \\
- Duration is too short; depressed moods can develop over months
\end{tabular}

\subsection{Critique of Critique of the Fermented Food Study}

\subsubsection{Study Design}

\subsubsection{Sample Size}

The final sample size includes 710 students (445 female). Although the sample size is large enough compared with the other two studies, it lacks diversity as the samples are all students from the same school and participated in the same course.

\subsubsection{Inclusion Criteria and Exclusive Criteria}

Data were collected as part of mass testing for introductory psychology classes at a medium-size public liberal arts university in Virginia, which counted for a partial fulfillment of a course requirement ${ }^{[12]}$.
22 students (The original number was 732) were excluded due to missing data on key variables of interest. This is a potential justified reason for exclusion because these individuals did not provide the necessary critical information for follow-up and data analysis.

\subsubsection{Measurements}

The researchers measured participants' social anxiety and neuroticism by two well-known scales (SPAI-23 and Big Five Personality Inventory, as shown in Figure 1). In addition, the effects of exercise and fermented food on social anxiety were analyzed by measuring the Exercise Frequency and Food Frequency after assigning them. All surveys were completed electronically using Qualtrics software, and the setting of the topic is relatively reasonable and comprehensive.

Table 9 Two Scales in this Study ${ }^{[13]}$

\begin{tabular}{|c|c|}
\hline Type of the survey & Explanation and Evaluation \\
\hline $\begin{array}{l}\text { Social Phobia and } \\
\text { Anxiety Inventory } \\
\text { (SPAI-23) }\end{array}$ & $\begin{array}{l}\text { Participants respond using a } 5 \text {-point scale ranging from } 0 \text { (never) to } 4 \text { (always) to indicate how } \\
\text { frequently they experience symptoms related to social phobia (16 items) and agoraphobia ( } 7 \\
\text { items). This is a fairly authoritative measure. }\end{array}$ \\
\hline $\begin{array}{l}\text { Big } \\
\text { Personality } \\
\text { Inventory }\end{array}$ & $\begin{array}{l}\text { Selecting } 8 \text { items of the } 44 \text {-item Big Five Inventory assess the personality traits extraversion, } \\
\text { agreeableness, conscientiousness, openness, and neuroticism (John and Srivastava, 1999). } \\
\text { Participants respond using a } 5 \text {-point scale ranging from } 1 \text { (strongly disagree) to } 5 \text { (strongly } \\
\text { agree) to indicate how much a given characteristic applies to them. This is also a relatively } \\
\text { authoritative measure. }\end{array}$ \\
\hline $\begin{array}{l}\text { Exercise } \\
\text { Frequency }\end{array}$ & $\begin{array}{l}\text { Frequency of movement was assessed by a list of questions of how long and how often they } \\
\text { exercise, with different scores given to different responses. However, it is doubtful that the } \\
\text { participants can keep accurate track of their exercise frequency. This design is prone to } \\
\text { reporting bias. }\end{array}$ \\
\hline Food Frequency & $\begin{array}{l}\text { Measurements of participants' food frequency were similar to exercise frequency, but were } \\
\text { more complex. Participants were first asked to recall the types of foods they had consumed over } \\
\text { the past } 30 \text { days, then to rate how often they ate those foods on a seven-point scale and assign } \\
\text { scores to them. }\end{array}$ \\
\hline
\end{tabular}




\subsubsection{Risk of biases (Table 10 shows)}

Table 10 Risk of biases

\begin{tabular}{|l|l|}
\hline Type of bias & Explanation \\
\hline $\begin{array}{l}\text { Reporting bias and Recall } \\
\text { bias }\end{array}$ & $\begin{array}{l}\text { This is mainly caused by the subjectivity of questionnaire filling. The experimental } \\
\text { data are all from four subjectivity scales. } \\
\text { Firstly, participation may selectively disclose or withhold information which will lead } \\
\text { to reporting bias. Secondly, recall bias camm from participants' inability to } \\
\text { accurately remember past experiences and details. }\end{array}$ \\
\hline Selection bias & $\begin{array}{l}\text { No randomized trial was used, and the researchers only selected a sample of } \\
\text { students from the same university participating in the same course, which lacks } \\
\text { universality and reproducibility. }\end{array}$ \\
\hline
\end{tabular}

\subsubsection{Presentation of the Results}

To establish the first part of the results, the researchers used multiple regression data to statistically analyze positive and negative associations between neuroticism, fermented foods and exercise and social anxiety.

To further explore the relationship between neuroticism and fermented food, the researchers looked at a statistically significant interaction between neuroticism and fermented food consumption. They visualized the relationship between the two.

Overall, the results are clear. The regression analysis table clearly shows three pairs of relationships: a) Neuroticism was positively correlated with social anxiety, with those high on trait neuroticism having higher levels of social anxiety. b) Fermented food consumption was negatively correlated with social anxiety indicating that those who consume more fermented foods have lower levels of social anxiety. c) Both fruit and vegetable consumption and exercise frequency were negatively correlated with social anxiety. ${ }^{[14]}$ In addition, the visualized graph clearly demonstrated the effect of fermented food on neuroticism. However, the researchers used a qualitative instead of quantitative approach ("high" and "low") to show the relationship between the two variables (as shown in Figure 2). This design lacks precision.

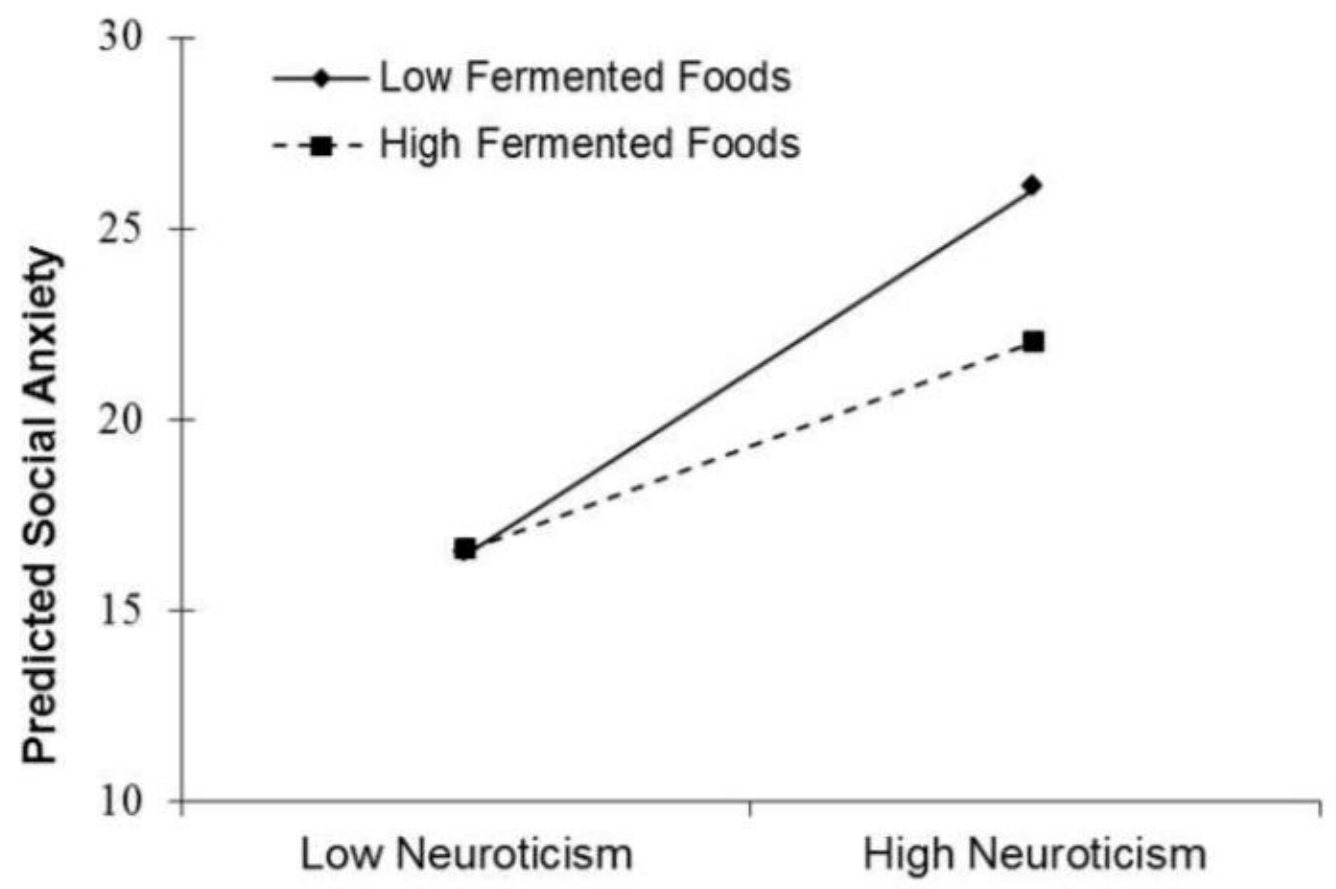

Figure 2 The table extracted from the study showing the results [15] 


\subsubsection{Strengthens and Limitations (Table 11 shows)}

Table 11 Risk of biases

\begin{tabular}{|l|l|}
\hline Strengthens & $\begin{array}{l}\text { - The paper is the first to demonstrate that consumption of fermented foods } \\
\text { interacting with neuroticism to predict social anxiety symptoms. } \\
\text { - These findings are consistent with results from previous preclinical. }\end{array}$ \\
\hline Limitations & $\begin{array}{l}\text { - } \\
\text { - } \text { - Uneasuremente of selection and reporting bias } \\
\text { - Lack of a more diverse sample of participants and subgroup Settings } \\
\text { - Neglect of antidepressants, anti-anxiety medications, probiotic pills and } \\
\text { supplements }\end{array}$ \\
\hline
\end{tabular}

\section{DISCUSSION}

\subsection{Discussion of the Media Article}

This work selected 3 statements from the media article to analyze in depth. The three statements are presented in the table below along with the appraisal for their credibility and possible improvements. (Table 12 shows)

Table 12 The text analysis

\begin{tabular}{|c|c|}
\hline $\begin{array}{l}\text { Relevant Quotes from the Media } \\
\text { Article }\end{array}$ & Evaluation \\
\hline $\begin{array}{l}\text { "Consume more vitamin C } \\
\text { Foods such as red and green peppers, } \\
\text { oranges, grapefruit and kiwi are rich in } \\
\text { vitamin C, which in high doses has } \\
\text { antidepressant effects and can } \\
\text { improve mood, and may be helpful in } \\
\text { treating stress-related disorders. } \\
\text { Other research has revealed that } \\
\text { vitamin C may help reduce anxiety } \\
\text { among high school students. } \\
\text { To boost your vitamin C intake, aim to } \\
\text { include one vitamin C-rich food with a } \\
\text { meal, and another for a snack. You } \\
\text { could also try one of my favorites: } \\
\text { dark chocolate-dipped kiwis or } \\
\text { oranges for dessert!"[16] }\end{array}$ & $\begin{array}{l}\text { The media article referred to the journal article about the effect of vitamin } \\
\text { C on anxiety in the sentence "Other Research has revealed that Vitamin C } \\
\text { may help reduce anxiety among high school students". The only problem } \\
\text { with this reference is the phrase"among high school students". After } \\
\text { carefully reading the journal article, this work noticed that the sample the } \\
\text { study selected have high baseline BAl score, indicating their high level of } \\
\text { anxiety, due to the study's negligence to its own exclusion criterion. The } \\
\text { "high school students" population, by itself, should indicate people } \\
\text { without psychiatric or clinical conditions, while the pariticipants in the } \\
\text { study have such conditions. A result of this would be that the study result } \\
\text { extracted from the sample in the study cannot be generalized to the "high } \\
\text { school students" population. This problem would be eliminated if the } \\
\text { author of the media article did read over the journal article and notice the } \\
\text { inconsistency between its exclusion criterion and its samples' baseline BAl } \\
\text { scores. A possible improvement would be: "Other Research has revealed } \\
\text { that Vitamin C may help reduce anxiety among high school students } \\
\text { suffering from anxiety." } \\
\text { Another option could be not to cite this research, as it has some major } \\
\text { flaws and inclarities in its procedure. }\end{array}$ \\
\hline
\end{tabular}




\begin{tabular}{|c|c|}
\hline $\begin{array}{l}\text { "Fill up on fermented foods } \\
\text { Fermented foods such as yogurt, } \\
\text { kombucha, kefir, tempeh and } \\
\text { sauerkraut contain friendly bacteria } \\
\text { known as probiotics, which have the } \\
\text { ability to reduce stress and cortisol } \\
\text { levels. } \\
\text { In fact, randomized controlled trials } \\
\text { featuring probiotics suggest a causal } \\
\text { link between the gut microbiota and } \\
\text { stress responding. } \\
\text { Feeling shy? Fermented foods may } \\
\text { help reduce symptoms of social } \\
\text { anxiety, too, research has shown. } \\
\text { These probiotic- rich foods may also } \\
\text { help control negative thoughts that } \\
\text { are associated with low moods." [17] }\end{array}$ & $\begin{array}{l}\text { The observational study on the fermented food was used as the source for } \\
\text { the media article in the sentence "Fermented foods may help reduce } \\
\text { symptoms of social anxiety". The interaction model demonstrated in the } \\
\text { journal article found that high consumption of fermented foods was } \\
\text { associated with fewer symptoms of social anxiety. Therefore, the effect of } \\
\text { fermented foods on reducing social anxiety can be verified to some } \\
\text { extent. However, the study itself has deficiencies. On the one hand, in the } \\
\text { selection of research samples, the age distribution of the participants is } \\
\text { between the ages of } 18 \text { and } 38, \text { so this result cannot be generalized to all } \\
\text { generations. On the other hand, in the study, the researchers could not } \\
\text { rule out the presence of antidepressants or anti-anxiety medications or } \\
\text { the use of probiotic pills and supplements, so the effectiveness of } \\
\text { fermented foods in reducing social anxiety is also questionable. The } \\
\text { suggestion is that the suggestion given by media article should be } \\
\text { supplemented by references and quotation to other journal articles that } \\
\text { can complement the above two points. }\end{array}$ \\
\hline
\end{tabular}

\subsection{Quality of the Journal Articles}

The two randomized controlled trials this work analyzed both have a sample size that is too small to be representative. This problem can result in the low statistical power of the studies and thus a high likelihood for getting false positive results. Moreover, the study durations for both studies are too short. They only inspected the short-term effects of the treatment while neglecting the often more important, long term effects. However, this work does acknowledge that recruiting more participants for longer time can generate higher cost for the research institutes.

Through comparing the study about Vitamin $\mathrm{C}$ and the one about probiotics, this work derived a suggestion for a problem that should be addressed in future studies. In the Vitamin $\mathrm{C}$ article, the study did not include any specific information about the pretrial selection. The readers have no idea how the inclusion and exclusion criterion are implemented. It leaves the study vulnerable when the readers spot the possible invalidity of the criterion later in the study, like what this work did. On the contrary, in the probiotics article included a very detailed explanation about how they manged the pre-trial selection to ensure that all participants are tested against the inclusion and exclusion criterion. For example, inventories are given to potential participants before the trial to test whether their psychiatric conditions are suitable for the treatment. The suggestion in this paper for future studies is to always include a clear methodology for the pre-trial selection. As indicated by the article about vitamin $\mathrm{C}$, the method for the pre-trial selection might often be neglected in clinical trials as the studies tend to focus more on the methodologies for the treatments and the result analysis.

Additionally, the article on fermented food is analyzed from another point of view as it is an observational study instead of a RCT. The experiment was convincing to a certain extent, with $1 / 2$ of the selected measurement scale being authoritative (SPAI-23 and Big Five Personality Inventory) and the rest being well considered. The sample size is also not small, with 710 particpants. However, due to the absence of a randomized trial, this experiment was temporarily unable to exclude some influencing factors, and the questionnaire survey on the subjects was also full of contingency. As mentioned above, there are many limitations. Therefore, further clinical trial data is needed to make the article more convincing.

\subsection{Policy Context}

The media article included up to six different kinds of food that reduces stress, which are a large amount of information that lacks fact checking. Therefore, their readers should note that no valid policy suggestions could be evidently deducted from this article alone. As for the journal articles, more repetition and improvement of their studies is needed before further conclusions could be gain on how vitamin $\mathrm{C}$, probiotics and fermented foods intake could lower stress level in a practical sense. There also lacks policy on how stress level could be quantitatively measured and accessed in researches targeting stress relieve.

\section{CONCLUSION}

Overall, through systematic qualitative and critical analysis, the media articles found in this work did not 
distort the findings in journal articles. Some readers suffering from anxiety disorders can find a relatively effective way of food therapy through the suggestions given by this media article. However, media article to some extent ignored the quality of the studies cited. For example, this paper hs found several common problems in these studies, including limited sample size, the existence of selection bias, and unclear trial design. The media article would be prone to misinformation once its claims are based on poorly conducted research.

This work also has some inadequacies. This work only selects a few sentences from media articles for analysis, which may lead to incomplete evaluation. Moreover, even though this work derived a methodology to synchronize the analysis in this work, This work is still writing each critique separately. A problem for that would be the inconsistent presentation of the findings in the paper. This problem also arises from the diverse characteristics, strengths, and shortages of the studies we analyze. Different paper may have different problems that this work wants to emphasize on. It is difficult to keep a unified style for these reasons.

\section{REFERENCES}

[1] Gonzalez, M. J., Miranda-Massari, J. R. (2014) Diet and stress. The Psychiatric clinics of North America, 37(4): 579-589.

[2] Aucoin, M., Bhardwaj, S. (2016) Generalized Anxiety Disorder and Hypoglycemia Symptoms Improved with Diet Modification. Case reports in psychiatry, 2016: 7165425.

[3] Gonzalez, M. J., Miranda-Massari, J. R. (2014) Diet and stress. The Psychiatric clinics of North America, 37(4): 579-589.

[4] Opie, R., O'Neil, A., Itsiopoulos, C., Jacka, F. (2015) The impact of whole-of-diet interventions on depression and anxiety: a systematic review of randomised controlled trials. Public Health Nutrition, 18: 2074 - 2093.

[5] Lai, J. S., Hiles, S., Bisquera, A., Hure, A. J., McEvoy, M., Attia, J. (2014) A systematic review and meta-analysis of dietary patterns and depression in community-dwelling adults. The American journal of clinical nutrition, 99(1): 181197.

[6-9] de Oliveira, I. J., de Souza, V. V., Motta, V., Da-Silva, S. L. (2015) Effects of Oral Vitamin C Supplementation on Anxiety in Students: A Double-Blind, Randomized, Placebo-Controlled Trial. Pakistan journal of biological sciences: PJBS, 18(1): 11-18.

[10] Steenbergen, L., Sellaro, R., van Hemert, S., Bosch,
J. A., Colzato, L. S. (2015) A randomized controlled trial to test the effect of multispecies probiotics on cognitive reactivity to sad mood. Brain, behavior, and immunity, 48: 258-264.

[11] Sheehan, D. V., Lecrubier, Y., Sheehan, K. H., Amorim, P., Janavs, J., Weiller, E., Hergueta, T., Baker, R., Dunbar, G. C. (1998) The Mini-International Neuropsychiatric Interview (M.I.N.I.): the development and validation of a structured diagnostic psychiatric interview for DSM-IV and ICD-10. The Journal of clinical psychiatry, 59 Suppl 20: 22-57.

[12-15] Hilimire, M. R., DeVylder, J. E., Forestell, C. A. (2015). Fermented foods, neuroticism, and social anxiety: An interaction model. Psychiatry research, 228(2): 203-208.

[16-17] Lisa Drayer.(2021) Foods that can reduce stress. https://edition.cnn.com/2021/01/23/health/stress-re lieving-foods-wellness/index.html 